SLAC-PUB-12858

October 2007

\title{
Steady State Analysis of Short-wavelength, High-gain FELs in a Large Storage Ring *
}

\author{
Z. Huang, K. Bane, Y. Cai, A. Chao, R. Hettel \\ Stanford Linear Accelerator Center, Menlo Park, CA 94025 \\ C. Pellegrini \\ University of California at Los Angeles, Los Angeles, CA 90095
}

\begin{abstract}
Storage ring FELs have operated successfully in the low-gain regime using optical cavities. Discussions of a high-gain FEL in a storage ring typically involve a special bypass to decouple the FEL interaction from the storage ring dynamics. In this paper, we investigate the coupled dynamics of a high-gain FEL in a large storage ring such as PEP and analyze the equilibrium solution. We show that an FEL in the EUV and soft x-ray regimes can be integrated into a very bright storage ring and potentially provides three orders of magnitude improvement in the average brightness at these radiation wavelengths. We also discuss possibilities of seeding with HHG sources to obtain ultra-short, high-peak power EUV and soft x-ray pulses.
\end{abstract}

Presented at International Workshop on Frontiers in FEL Physics and Related Topics (Elba Island, Italy, September 8 - 14, 2007)

and submitted to Nuclear Instruments and Methods in Physics A

*Work supported by Department of Energy contract DE-AC02-76SF00515. 


\title{
Steady state analysis of short-wavelength, high-gain FELs in a large storage ring
}

\author{
Z. Huang, K. Bane, Y. Cai, A. Chao, R. Hettel \\ Stanford Linear Accelerator Center, Stanford, CA 94309 \\ C. Pellegrini \\ University of California at Los Angeles, Los Angeles, CA 90095
}

\begin{abstract}
Storage ring FELs have operated successfully in the low-gain regime using optical cavities. Discussions of a highgain FEL in a storage ring typically involve a special bypass to decouple the FEL interaction from the storage ring dynamics. In this paper, we investigate the coupled dynamics of a high-gain FEL in a large storage ring such as PEP and analyze the equilibrium solution. We show that an FEL in the EUV and soft x-ray regimes can be integrated into a very bright storage ring and potentially provides three orders of magnitude improvement in the average brightness at these radiation wavelengths. We also discuss possibilities of seeding with HHG sources to obtain ultra-short, highpeak power EUV and soft x-ray pulses.
\end{abstract}

\section{Introduction}

Storage ring free-electron lasers (FELs) have operated successfully in the low-gain regime using optical cavities. The shortest wavelength achieved by storage ring FELs is about $200 \mathrm{~nm}[1,2]$, limited by mirror reflectivity. Discussions of mirrorless, highgain FELs in a storage ring typically involve a special bypass to provide the FEL interaction about once every damping time and hence decouple the FEL from the storage ring dynamics (see, e.g., Refs. [3$5]$ ). However, a storage ring FEL with a bypass line has never been built due to the rapid development in linac-based, short-wavelength FELs.

Nevertheless, storage rings offer very reliable and stable electron beams at high repetition rates. In a large storage ring with long straight sections such as PEP, a new design with an ultra-low emittance lattice brings the beam brightness to a level sufficient to drive a short-wavelength, high-gain FEL [6]. In this paper, we investigate the coupled dynamics of a high-gain FEL in such a storage ring without a bypass. In Sec. 2, we study the effect of energy spread on a high-gain FEL and the induced energy spread during the lasing process using a one-dimensional (1D) model. The coupled FELring dynamics is solved in this model to obtain the equilibrium solution for the energy spread and the radiation power. In Sec. 3, we briefly discuss lattice design and beam dynamics issues of using PEP as an ultra-low emittance, high-current light source. In Sec. 4, we analyze the steady-state performance of a self-amplified spontaneous emission (SASE) FEL and show its potential enhancement over the spontaneous radiation in the extreme ultraviolet (EUV) and soft x-ray wavelength regime. Such an FEL may also be seeded with higher-harmonic generation (HHG) sources to obtain ultra-short (10-50 fs), high-peak power photon pulses. 


\section{1D steady-state analysis}

\subsection{Energy spread in a high-gain FEL}

We first consider a 1D FEL model and neglect three-dimensional (3D) effects. For a beam with a Gaussian energy spread of rms width $\sigma_{\delta}$ entering a planar undulator, the 1-D power gain length is approximately

$$
L_{G} \approx L_{G 0}\left(1+\sigma^{2}\right) .
$$

Here $L_{G 0}=\lambda_{u} /(4 \pi \sqrt{3} \rho)$ is the $1 \mathrm{D}$ gain length for a beam with vanishing energy spread, $\lambda_{u}$ is the undulator period, $\sigma \equiv \sigma_{\delta} / \rho, \rho$ is the FEL Pierce parameter [7]

$$
\rho=\left[\frac{1}{8 \pi} \frac{\hat{I}}{I_{A}}\left(\frac{K[\mathrm{JJ}]}{1+K^{2} / 2}\right)^{2} \frac{\gamma \lambda_{r}^{2}}{\left(\pi \sigma_{x}^{2}\right)}\right]^{1 / 3},
$$

with $\hat{I}$ the bunch peak current, $I_{A}=e c / r_{e} \approx 17$ $\mathrm{kA}$ the Alfvén current, $K$ the undulator parameter, [JJ] the usual Bessel function factor associated with a planar undulator, $\gamma$ the beam energy in units of $m c^{2}, \lambda_{r}$ the FEL resonant wavelength, and $\sigma_{x}$ the rms transverse beam size.

It can be shown that the beam energy spread increases during the high-gain process according to

$$
\Delta\left(\sigma^{2}\right)_{F E L} \approx 2 \frac{P}{\rho P_{\text {beam }}} .
$$

where $P$ is the radiation power and $P_{\text {beam }}$ is electron beam power $P_{\text {sat }}$. Throughout this paper, we refer power to its peak value unless average power is explicitly stated.

\subsection{Equilibrium solution}

For a short-wavelength FEL, the FEL-induced microbunching is washed out in a fraction of one turn because

$$
k_{r} R_{56} \sigma_{\delta} \gg 1,
$$

where $k_{r}=2 \pi / \lambda_{r}, R_{56}=\alpha_{c} c T_{0}, T_{0}$ is the revolution time, and $\alpha_{c}$ is the momentum compaction of the ring. The only memory in the beam due to the FEL interaction is the increased energy spread, which must be properly taken into account in addition to radiation damping and quantum excitation.

The longitudinal dynamics can be modeled as

$$
\frac{d \sigma^{2}}{d t}=\frac{\sigma_{0}^{2}-\sigma^{2}}{\tau_{s}}+\frac{\Delta\left(\sigma^{2}\right)_{F E L}}{T_{0}},
$$

where we have assumed only one FEL undulator in the ring, $t$ is the time variable, $\tau_{s}$ is the longitudinal damping time of the ring, and $\sigma_{\delta 0} \equiv \sigma_{0} \rho$ is the equilibrium energy spread in the absence of the FEL interaction. Defining $N_{d}=\tau_{s} / T_{0}$ as number of turns for the longitudinal damping time and $n=t / T_{0}$, we have

$$
\frac{d \sigma^{2}}{d n}=\frac{\sigma_{0}^{2}-\sigma^{2}}{N_{d}}+\frac{2 P}{\rho P_{\text {beam }}} .
$$

For a SASE FEL starting up from electron shot noise, the radiation power can be written as $[8,9]$

$$
P \approx \frac{1}{9} P_{n} \exp \left(\frac{z}{L_{G}}\right)
$$

where

$$
P_{n} \approx \sqrt{2 \pi} \frac{\rho^{2} \gamma m c^{3}}{\lambda_{r}}
$$

is the effective noise power.

When the equilibrium energy spread increases due to the FEL interaction, the bunch will lengthen and the peak current $\hat{I}$ will be reduced. Since the FEL parameter $\rho \propto \hat{I}^{1 / 3}$ as shown in Eq. (2), we neglect this weak dependence here and use Eq. (1) with a constant $\rho$ to estimate the effect of energy spread on the FEL gain length. (In Sec. 4, however, the current dependence on the energy spread is included self-consistently in the numerical calculations.)

The equilibrium energy spread $\sigma_{e}$ can be found by setting $d \sigma_{e}^{2} / d n=0$. For a relatively short undulator, the FEL induced energy spread is much smaller than the quantum excitation level per turn $\left(\sim \sigma_{e}^{2} / N_{d}\right)$, so we can drop the last term in Eq. (6) and obtain the trivial equilibrium solution

$$
\sigma_{e}=\sigma_{0}, \quad P_{e}=\frac{1}{9} P_{n} \exp \left[\frac{z}{L_{G}\left(\sigma_{0}\right)}\right] .
$$

For a longer undulator (than a characteristic distance $z_{0}$ to be determined), we may assume the the square of equilibrium energy spread increases linearly with the undulator distance $z$ as

$$
\sigma_{e}^{2}=\sigma_{0}^{2}+a\left(z-z_{0}\right)
$$

and use the approximation

$$
\exp \left[\frac{z}{L_{G}(\sigma)}\right] \approx \exp \left(\frac{1}{L_{G 0} a}-\frac{b}{z}\right),
$$

where $b=\left(1+\sigma_{0}^{2}-a z_{0}\right) /\left(L_{G 0} a^{2}\right)$. For the parameter regime of interest (i.e. for $0.3<\frac{z}{b}<1$ ), we write

$$
\exp \left(-\frac{b}{z}\right) \approx-0.1+0.5 \frac{z}{b} \text {. }
$$


The self-consistent solution is given by

$$
\begin{aligned}
0.1 \frac{2 P_{n} N_{d}}{9 \rho P_{\text {beam }}} \exp \left(\frac{1}{L_{G 0} a}\right) & \approx a z_{0}, \\
0.5 \frac{2 P_{n} N_{d}}{9 \rho P_{\text {beam }} b} & =a,
\end{aligned}
$$

which can be solved for $a, b$ and $z_{0}$. Thus, at undulator distance $z>z_{0}$, the equilibrium energy spread increases according to Eq. (10), and the equilibrium FEL power becomes

$$
P_{e}(z)=\frac{\rho P_{\text {beam }}}{2 N_{d}}\left(\sigma_{e}^{2}-\sigma_{0}^{2}\right)=\frac{\rho P_{\text {beam }}}{2 N_{d}} a\left(z-z_{0}\right) .
$$

The transition from Eq. (9) to Eq. (14) occurs at about $z_{0}$, the distance within the undulator where the induced FEL energy spread is a significant fraction of the quantum excitation level.

For a sufficiently long undulator, a rough estimate of the maximum equilibrium power may be obtained by equating the energy diffusion from the FEL interaction to the energy diffusion from quantum excitation

$$
P_{\text {max }} \sim \rho P_{\text {beam }} \frac{\sigma_{0}^{2}}{N_{d}}=P_{\text {beam }} \frac{\sigma_{\delta 0}^{2}}{\rho N_{d}} .
$$

For example, if $\sigma_{\delta 0}=10^{-3}, N_{d}=10^{3}$, and $\rho=$ $10^{-3}$, then $P_{\text {max }} \sim 10^{-6} P_{\text {beam }}$. Comparing this to the FEL saturation power $P_{\text {sat }} \sim \rho P_{\text {beam }} \sim$ $10^{-3} P_{\text {beam }}$, the maximum equilibrium power is three orders of magnitude below saturation. Nevertheless, considering that the FEL gain is typically five to six orders of magnitude above the shot noise level, the equilibrium power is still two to three orders of magnitude above the spontaneous radiation and may accordingly increase the average brightness.

\section{PEP as a high-brightness light source}

\subsection{Ultra-low emittance lattice}

Currently, the PEP tunnel contains two rings of a hexagon shape. The circumference of the rings is 2.2 kilometers, including 1.5 kilometers arcs and six long straight sections (each more than $100 \mathrm{~m}$ in length). Since the bending magnets in the arcs mostly determine the equilibrium emittance due to synchrotron radiation, a ring in the $\mathrm{PEP}$ tunnel is effectively equivalent to, from the point viewed of minimum emittance, a 1.5-kilometers circular synchrotron facility. The Theoretical Minimum Emittance (TME) lattice can achieve an emittance three times smaller than double-bend achromat (DBA) lattice for the same bending angle. Since PEP already has six long straight sections, the easiest way to make a low emittance lattice is to pack the arcs with as many TME cells as possible having weaker bending magnets. A detailed lattice design that uses damping wigglers to achieve a lower emittance can be found in Ref. [6].

One set of possible parameters for a new PEP lattice is given in Table 1. One sees that the horizontal betatron tune is extremely high because of the strong focusing that is needed to make the ultra-low emittance. The energy spread and damping time are largely determined by the damping wigglers (total length $\sim 150 \mathrm{~m}$ ). The RF frequency used in the calculation is $500 \mathrm{MHz}$.

Table 1

Parameters of an ultra-low emittance ring in the PEP tunnel.

\begin{tabular}{|l|c|c|}
\hline Description & no wigglers & with wigglers \\
\hline Beam energy $\gamma m c^{2}(\mathrm{GeV})$ & 4.5 & 4.5 \\
Emittance $\epsilon_{x}(\mathrm{~nm}-\mathrm{rad})$ & 0.10 & 0.05 \\
Damping time $\tau_{x}(\mathrm{~ms})$ & 177 & 15 \\
Horizontal tune $\nu_{x}$ & 88.57 & 99.57 \\
Vertical tune $\nu_{y}$ & 38.64 & 39.64 \\
Synchrotron tune $\nu_{s}$ & 0.0065 & 0.0087 \\
Mom. compaction $\alpha_{c}$ & $6.96 \times 10^{-5}$ & $6.86 \times 10^{-5}$ \\
Energy spread $\sigma_{\delta 0}$ & $3.9 \times 10^{-4}$ & $1.14 \times 10^{-3}$ \\
Natural chromaticities $\xi_{x}, \xi_{y}$ & $-143.4,-62.5$ & $-175.6,-72.4$ \\
Energy loss $U_{0}$ per turn $(\mathrm{MeV})$ & 0.37 & 4.34 \\
RF voltage $(\mathrm{MV})$ & 5 & 10 \\
rms bunch length $\sigma_{s 0}(\mathrm{~mm})$ & 1.45 & 3.13 \\
\hline
\end{tabular}

\subsection{High peak current studies}

Since high peak current is essential for FEL applications, we study the single bunch current effects in such a low emittance PEP ring. The threshold current is typically determined by the microwave instability at wavelengths shorter than the natural bunch length. At millimeter wavelengths or below, the CSR impedance in the dipoles dominates over other impedance sources. The CSR instability threshold for a coasting beam in free space is [10]

$$
\frac{\hat{I}_{t h}}{I_{A}}=\frac{\gamma \alpha_{c} \sigma_{\delta 0}^{2} c T_{0}}{2\left(\pi R \lambda^{2}\right)^{1 / 3}}
$$

$\hat{I}_{t h}$ is the threshold peak current, and $R=98 \mathrm{~m}$ is the dipole bending radius of the TME lattice. Thus, 
the threshold current is determined by the longest wavelength before vacuum chamber shielding takes effect. Using a parallel plate model, the shielding cutoff wavelength can be determined as [11]

$$
\lambda_{s}=\frac{2^{5 / 2} g^{3 / 2}}{\sqrt{\pi} R^{1 / 2}},
$$

where $g$ is the half height of the vacuum chamber. Taking $g=2 \mathrm{~cm}$ for a typical vacuum chamber, we find that the shielding cut-off wavelength is about $0.9 \mathrm{~mm}$.

Using $\lambda=\lambda_{s}$ in Eq. (16), we can estimate the threshold peak current as

$$
\frac{\hat{I}_{t h}}{I_{A}}=\frac{\gamma \alpha_{c} \sigma_{\delta}^{2} c T_{0}}{2^{8 / 3} g} .
$$

Since the threshold current scales quadratically with the beam energy spread, increasing the equilibrium energy spread in a storage ring within the FEL tolerance is more efficient for FEL gain than using a bunch compression scheme (where the current scales linearly with the energy spread). Thus, a ring with damping wigglers (having a larger energy spread and a lower transverse emittance) is more suitable for FEL operations than a ring without wigglers. In addition, the damping wigglers increase the energy diffusion due to quantum excitation and allow for a higher equilibrium FEL power as indicated in Eq. (15). For these reasons, the beam parameters with damping wigglers are adopted for the following FEL studies. The threshold peak current using Eq. (18) is estimated to be $230 \mathrm{~A}$. We assume that the lattice can be adjusted slightly to accommodate a more aggressive peak current of $300 \mathrm{~A}(1 \mathrm{~mA}$ average current). The total charge stored per bunch (with $3 \mathrm{~mm}$ rms bunch length) is $7.5 \mathrm{nC}$. More studies on the microbunching instability driven by CSR with realistic vacuum chamber geometry are necessary to determine the threshold current more accurately.

At such a high bunch density, intrabeam scattering (IBS) is not negligible. Detailed studies of IBS have been carried out for these ring parameters [6]. In Fig. 1 we plot, for the three values of the coupling parameter $\kappa$, the horizontal emittance as function of current. At $4.5 \mathrm{GeV}, 300 \mathrm{~A}$ and full coupling both transverse emittances are increased by a factor of 2.8 (compared to the natural emittances of $0.05 / 2=0.025 \mathrm{~nm}$ ), and the energy spread is increased by $4 \%$ over the nominal value. Since full coupling yields the smallest horizontal emittance, and a beam with equal transverse emittances is typically

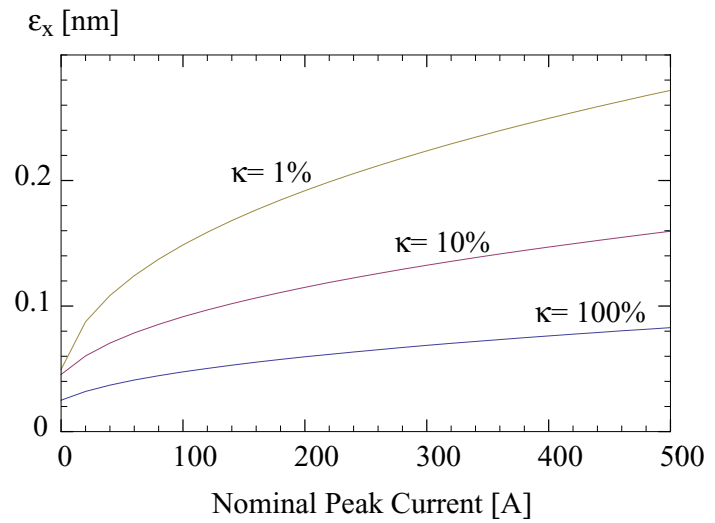

Fig. 1. The horizontal emittance $\epsilon_{x}$ vs. (nominal) peak current $\hat{I}$, for three values of coupling parameter $\kappa$.

preferred for FEL operations, we use the full coupling case for the following FEL studies.

\section{Short-wavelength FELs on PEP}

In this section, we discuss both SASE and seeded FEL studies when a long undulator $(\sim 100 \mathrm{~m})$ is integrated in one of the straight sections of the PEP ring.

\subsection{Steady-State SASE}

To incorporate the 3D effects, the 1D analysis of Sec. 2 is modified as follows. First, the $3 \mathrm{D}$ gain length $L_{G}^{3 D}$ dependence on the energy spread is modeled with Xie's fitting formula [12], and the effect of the energy spread on the peak current is also included (i.e., by taking $\hat{I} \propto \sigma_{\delta}^{-1}$ ). Secondly, for a beam with a relatively large energy spread, the SASE start-up process (i.e., Eq. (7)) should be modified to [13]

$$
P \approx P_{n} \exp \left(\frac{z}{L_{G}^{3 D}}\right),
$$

where $P_{n}$ is still given by Eq. (8). Lastly, we find that Eq. (3) is approximately valid in the $3 \mathrm{D}$ case according to FEL simulations. Equation (6) can now be solved numerically for a given set of ring and FEL parameters.

As a numerical example, we discuss a soft $\mathrm{x}$-ray FEL at $\lambda_{r}=3.3 \mathrm{~nm}$ using $\lambda_{u}=5 \mathrm{~cm}$ and $\bar{\beta}=$ $4 \mathrm{~m}$ (see Table 2). Based on the numerical solutions of Eq. (6), we calculate the equilibrium energy spread and the radiation power as a function of the planned undulator length (see Fig. 2). For an undulator length less than $50 \mathrm{~m}$, the FEL interaction induces negligible energy spread, and the 
Table 2

Parameters for a soft x-ray FEL using PEP.

\begin{tabular}{|l|c|}
\hline Description & Value \\
\hline Longitudinal damping time $\tau_{s}$ (with wigglers) & $7.5 \mathrm{msec}$ \\
normalized transverse emittance after IBS $\gamma \varepsilon_{x, y}$ & $0.6 \mu \mathrm{m}$ \\
peak current $\hat{I}_{e}$ (at equilibrium) & $270 \mathrm{~A}$ \\
rms energy spread $\sigma_{\delta e}$ (at equilibrium) & $1.26 \times 10^{-3}$ \\
undulator period $\lambda_{u}$ & $5 \mathrm{~cm}$ \\
undulator parameter & 4.3 \\
peak magnetic field & $0.92 \mathrm{~T}$ \\
average beta in undulator $\bar{\beta}$ & $4 \mathrm{~m}$ \\
active undulator length $z$ & $100 \mathrm{~m}$ \\
FEL wavelength $\lambda_{r}$ & $3.3 \mathrm{~nm}$ \\
FEL peak power (at equilibrium) & $\sim 200 \mathrm{~kW}$ \\
RMS pulse duration & $\sim 10 \mathrm{ps}$ \\
repetition rate (single bunch) & $136 \mathrm{kHz}$ \\
FEL average power (single bunch) & $\sim 0.7 \mathrm{~W}$ \\
\hline
\end{tabular}

FEL power grows exponentially with undulator distance. For a longer undulator, the equilibrium energy spread starts to increase as predicted in Sec. 2, up to $1.26 \times 10^{-3}$ in a $100-\mathrm{m}$ long undulator. The increased equilibrium energy spread lengthens the bunch slightly and reduces the peak current from $300 \mathrm{~A}$ to $270 \mathrm{~A}$. At equilibrium, the FEL peak power in the fundamental mode is about $200 \mathrm{~kW}$. As the SASE pulse length is comparable to the electron bunch length $(\mathrm{rms} \sim 10 \mathrm{ps})$, the average FEL power is about $0.7 \mathrm{~W}$ for a single electron bunch circulating in the ring (with about $1 \mathrm{~mA}$ average current).

To confirm these calculations, we performed GENESIS [14] simulation with $\hat{I}_{e}=270 \mathrm{~A}$ and $\sigma_{\delta e}=1.26 \times 10^{-3}$. Figure 3 shows that the SASE power reaches $500 \mathrm{~kW}$ at $100 \mathrm{~m}$, which includes contributions from the fundamental mode and higherorder transverse modes. Thus, the radiation at this stage of the exponential growth has not reached full transverse coherence. If needed, a monochromator can be used to select the fundamental mode just like the selection of the centrol cone radiation in a spontaneous source. This mode will be transversely coherent as the beam emittance is smaller than $\lambda_{r} /(4 \pi)$ and should have a power level of about $200 \mathrm{~kW}$. The spontaneous radiation power of 100 $\mathrm{m}$ undulator in a $0.1 \%$ bandwidth is estimated to be $1 \mathrm{~kW}$. Thus, the FEL gain is about a factor of 200 higher than the spontaneous level. We also performed a seeded FEL simulation at this wavelength

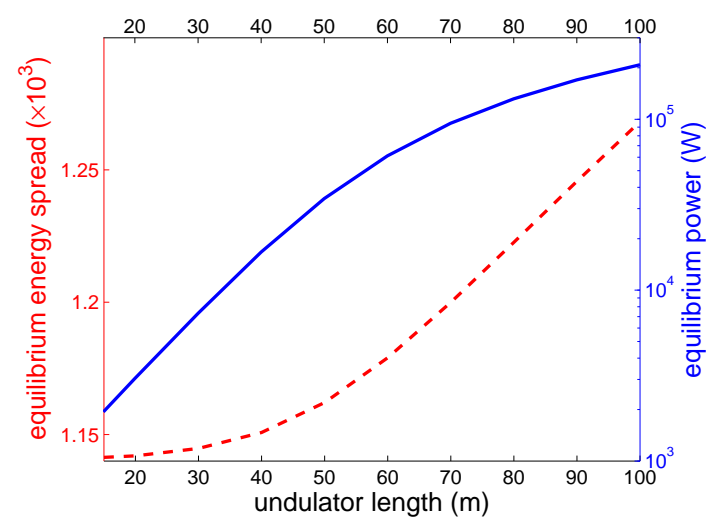

Fig. 2. Equilibrium energy spread (red dashed curve) and FEL power at $\lambda_{r}=3.3 \mathrm{~nm}$ (blue solid curve) as a function of the undulator length.

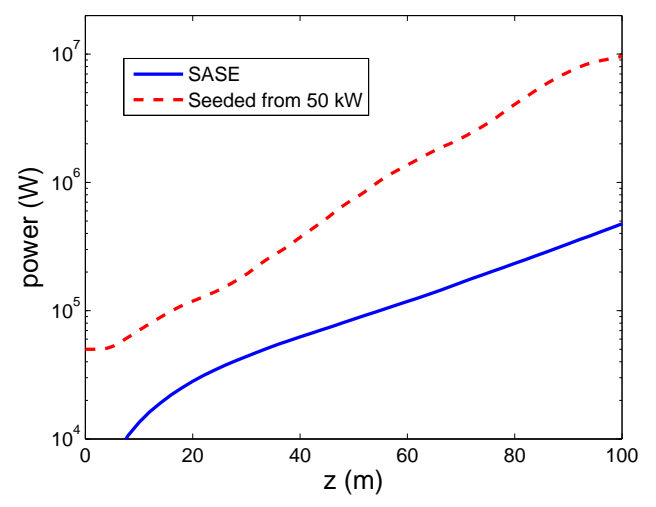

Fig. 3. Power evolution for a soft x-ray SASE FEL at $\lambda_{r}=3.3 \mathrm{~nm}$. For comparison, we also included a seeded FEL simulation (see Table 2 and discussions in Sec. 4.1).

and confirm a gain factor of 200 for a well-defined transverse mode (see Fig. 3). With a stored average current of about $1 \mathrm{~A}$ (about 1000 bunches in the ring), the average brightness will be enhanced by the same gain factor in the soft $\mathrm{x}$-ray regime, from $\sim 10^{22}$ to $\sim 10^{24}$ photons $/ \mathrm{sec} /(\mathrm{mm})^{2} /(\mathrm{mrad})^{2}$ in $0.1 \% \mathrm{BW}$, or about $10^{19}$ photons/sec within the FEL bandwidth $\left(\sim 3 \times 10^{-3}\right)$. Figure 4 shows the instantaneous power at $z=100 \mathrm{~m}$ for a small section (about 1\%) of the entire SASE pulse. There are more than 1000 temporal modes (spikes) in this pulse, so the statistical fluctuation of the radiated energy is less than 3\% for a single bunch, and less than $0.1 \%$ when averaged over 1000 bunches. 


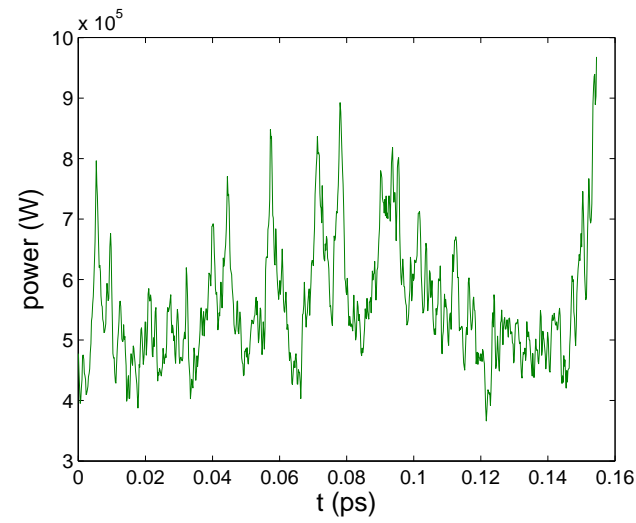

Fig. 4. Instantaneous power at $z=100 \mathrm{~m}$ for a small section (about 1\%) of the entire SASE pulse.

\subsection{Seeded FELs}

At longer radiation wavelengths $\left(\lambda_{r} \geq 10 \mathrm{~nm}\right)$, HHG sources with sufficient peak power may be used to seed the FEL process in order to bring it to the saturated power level. HHG sources are coherent both temporally and spatially, having pulse durations of about 10 to 50 fs [15]. Thus, seeding with an HHG source provides the possibility of generating fully coherent, femtosecond EUV and soft xray pulses, and has been demonstrated recently in a linac-based FEL [16]. We have conducted such simulations based on HHG sources at $30 \mathrm{~nm}$ and $10 \mathrm{~nm}$, with power levels achieved using present laser technology (albeit at very low repetition rates). Since seeding affects only a small portion of the electron beam at a low repetition rate, it has negligible impact on the beam dynamics of the ring. Thus, the equilibrium beam parameters can be determined by the steady-state SASE analysis as given above. At longer radiation wavelengths, the diffraction effect is stronger and, as a result, Xie's fitting formula does not model the gain length dependence on energy spread very accurately. Based on the simulation results for $\lambda_{r}=10$ and $30 \mathrm{~nm}$, we find that the equilibrium beam parameters at these longer wavelengths are similar to those given at $\lambda_{r}=3.3 \mathrm{~nm}$ (i.e., $\hat{I}_{e}=270 \mathrm{~A}$ and $\sigma_{\delta e}=1.26 \times 10^{-3}$ for a $100-\mathrm{m}$ undulator). Figure 5 shows both SASE and seeded simulations for a $\lambda_{r}=30 \mathrm{~nm}$ FEL using an undulator with $\lambda_{u}=10 \mathrm{~cm}$ and $\bar{\beta}=6 \mathrm{~m}$. The seeded FEL may reach saturation in $50 \mathrm{~m}$ if the seed power at the undulator entrance can be $10 \mathrm{MW}$. If this pulse is stretched by an optical grating in time to $500 \mathrm{fs}$ (from $50 \mathrm{fs}$ ) with a peak power at $1 \mathrm{MW}$, the seeded FEL can produce saturated radiation with a Fourier-

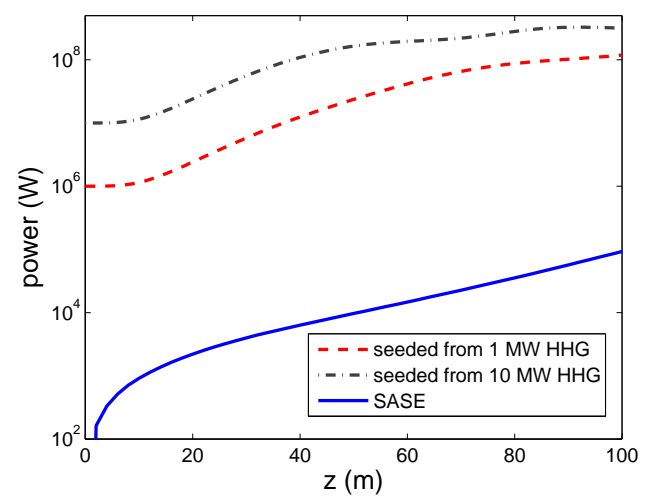

Fig. 5. Power evolution for SASE and seeded FELs at $\lambda_{r}=30 \mathrm{~nm}$ (see discussions in Sec. 4.2).

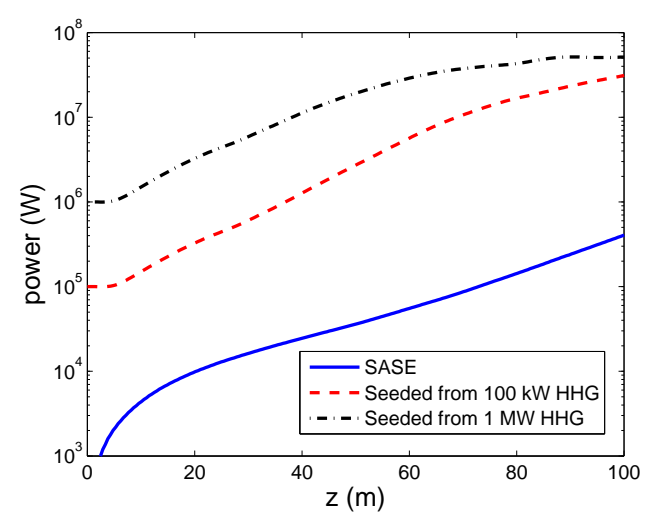

Fig. 6. Power evolution for SASE and seeded FELs at $\lambda_{r}=10 \mathrm{~nm}$ (see discussions in Sec. 4.2).

transform limited fwhm bandwidth of $1 \times 10^{-4}$.

Figure 6 shows a similar simulation study at $\lambda_{r}=$ $10 \mathrm{~nm}$, with a lower seed power but with a saturation length close to $100 \mathrm{~m}$ using an undulator with $\lambda_{u}=$ $6 \mathrm{~cm}$ and $\bar{\beta}=4 \mathrm{~m}$. In both 10 and $30 \mathrm{~nm}$ cases, we see that the SASE level reaches more than a hundred $\mathrm{kW}$ and is about three orders above the spontaneous power (about a few hundred $\mathrm{W}$ as photon energies are lower at these longer wavelengths).

\section{Discussion}

In this paper, we have studied the coupled dynamics of a long FEL undulator integrated into a large storage ring. For an ultra-low emittance, high peak current PEP ring, FEL exponential growth at EUV and soft x-ray wavelengths (from $50 \mathrm{~nm}$ down to about $3 \mathrm{~nm}$ ) can occur on a turn-by-turn basis, with only a modest degradation in beam quality. This analysis suggests that a bypass is not necessary 
for a short-wavelength, high-gain FEL in a storage ring and opens up the possibility of increasing the average brightness of a ring-based source by about two to three orders of magnitude over spontaneous source at these radiation wavelengths. The requirements on electron beam quality are demanding but lie within the limits of modern storage ring technology. With the development of short-wavelength HHG coherent sources, it is also possible to seed such an FEL to obtain femtosecond, high-peak power radiation pulses at the available seed repetition rate.

We note that placing a long undulator in a storage ring may have other beam dynamics consequences that can affect the ring operation. For instance, with a peak magnetic field on the order of $1 \mathrm{~T}$ for the 100-m long undulator, the effects of a spontaneous undulator radiation are almost equivalent to those of the damping wigglers for the ultra-low emittance PEP ring. Such a long undulator eliminates the requirement for a large number of the damping wigglers, but the remaining damping wigglers must be adjustable in their field strengths to compensate for the change of the spontaneous radiation level due to undulator gap variations (for FEL wavelength tuning). For a permanent-magnet undulator having 5$\mathrm{cm}$ in period, the full undulator gap is slightly above $1 \mathrm{~cm}$ to generate $\sim 1 \mathrm{~T}$ on-axis magnetic field. The resulting transverse resistive wall wakefield in a 100$\mathrm{m}$ small-gap vacuum chamber (or in the conducting image charge sheets for an in-vacuum undulator) may be a concern for multi-bunch instability. Preliminary estimates of the resistive wall instability for $1 \mathrm{~A}$ average current in this ring shows that it may be cured by a narrowband feedback system. Further studies are necessary to make a practical design of a high-gain FEL in such a large storage ring.

\section{Acknowledgments}

The authors would like to thank M.-E. Couprie, Y. Hao, X. Huang, I. Lindau, A. Merdji, and L.-H. $\mathrm{Yu}$ for many useful discussions. This work was supported by the U.S. Department of Energy contracts No. DE-AC02-76SF00515.

\section{References}

[1] V. Litvinenko et. al., Nucl. Instrum. Methods A 475, 195 (2001).

[2] M. Trovo et. al., Nucl. Instrum. Methods A 483, 157 (2002).
[3] J. Murphy and C. Pellegrini, Nucl. Instrum. Methods A 237, 159 (1985).

[4] K.-J. Kim et. al., Nucl. Instrum. Methods A 239, 54 (1985).

[5] H.-D. Nuhn et. al., Nucl. Instrum. Methods A 319, 89 (1992).

[6] R. Hettel et. al., PEP as a future light source report, 2007.

[7] R. Bonifacio, C. Pellegrini and L.M. Narducci, Opt. Commun. 50, 373 (1984).

[8] K.-J. Kim, Phys. Rev. Lett. 57, 1871 (1986).

[9] J.-M. Wang and L.-H. Yu, Nucl. Instrum. Methods A 250, 484 (1986).

[10] G. Stupakov and S. Heifets, Phys. Rev. ST Accel. Beams 5, 054402 (2002).

[11] R. Warnock, SLAC-PUB-5375 (1990).

[12] M. Xie, Proceedings of the 1995 Particle Accelerator Conference, 183 (1995).

[13] Z. Huang and K.-J. Kim, Nucl. Instrum. Methods A 475, 59 (2001).

[14] S. Reiche, Nucl. Instrum. Methods A 429, 243 (1999).

[15] A. Merdji, private communications.

[16] G. Lambert et. al., these proceedings. 